\title{
On the Generalized Mass Transport Equation to the Concept of Variable Fractional Derivative
}

\author{
Abdon Atangana ${ }^{1}$ and Adem Kilicman ${ }^{2}$ \\ ${ }^{1}$ Institute for Groundwater Studies, Faculty of Natural and Agricultural Sciences, University of the Free State, \\ Bloemfontein 9300, South Africa \\ ${ }^{2}$ Department of Mathematics and Institute for Mathematical Research, University Putra Malaysia, 43400 Serdang, Selangor, Malaysia
}

Correspondence should be addressed to Abdon Atangana; abdonatangana@yahoo.fr

Received 11 September 2013; Accepted 23 January 2014; Published 5 March 2014

Academic Editor: Necdet Bildik

Copyright (C) 2014 A. Atangana and A. Kilicman. This is an open access article distributed under the Creative Commons Attribution License, which permits unrestricted use, distribution, and reproduction in any medium, provided the original work is properly cited.

\begin{abstract}
The hydrodynamic dispersion equation was generalized using the concept of variational order derivative. The modified equation was numerically solved via the Crank-Nicholson scheme. The stability and convergence of the scheme in this case were presented. The numerical simulations showed that, the modified equation is more reliable in predicting the movement of pollution in the deformable aquifers, than the constant fractional and integer derivatives.
\end{abstract}

\section{Introduction}

Anomalous dispersion phenomena are systematically pragmatic in physics, chemistry, and biology fields [1-4]. To distinguish irregular diffusion phenomena, constant-order fractional diffusion equations were initiated and have received breathtaking success. Nonetheless, it has been recognized that the constant order fractional dispersion equations are not competent of typifying some multifaceted dispersion processes, for example, dispersion process in inhomogeneous or heterogeneous medium [5]. In addition, when we think about dispersion process in porous medium, if the medium structure or external field changes with time, in these circumstances, the constant-order fractional diffusion equation model cannot be used to well illustrate such phenomenon $[6,7]$. This is the case of the groundwater pollution problem; the medium through which the pollution is dispersing is heterogeneous and change with time. Motionless in some environmental science dispersion processes, the concentration of particles will determine the dispersion pattern $[8$, 9]. To solve the above problems, the variable-order (VO) fractional dispersion equation models have been suggested for use [10].

The leading edge work of VO operator can be traced to Samko et al. by introducing the variable order integration and
Riemann-Liouville derivative in [10]. It has been acknowledged as a prevailing modelling approach in the fields of viscoelasticity [10], viscoelastic deformation [11], viscous fluid [12], and anomalous transmission [13]. The problem encounter in groundwater is that the geometry of the aquifer in which the flow or the pollution takes place is not well known. In addition, the geological formation through which the contamination takes place changes in time and space. These phenomena cannot therefore accurately be described neither via the classical hydrodynamic dispersion equation nor via the fractional version of hydrodynamic dispersion equation. In this paper, we generalize the hydrodynamic dispersion equation using the concept of the variable order derivative.

\section{Definition and Problem Formulation}

The fractional operators (fractional derivatives and integrals) refer to the differential and integral operators of arbitrary order, and fractional differential equations refer to those containing fractional derivatives. The former are the generalization of integer-order differential and integral operators and the latter are the generalization of differential equations of integer order. The operators of variable-order, which fall into 
a more complex category, are the derivatives and integrals whose orders are the functions of certain variables.

\subsection{Variational Order Operator}

Definition 1. Left and right Riemann-Liouville integrals of variable order: let $0<\alpha(x, t)<1$ for all $(x, t) \in[a, b]$ and $f \in L_{1}[a, b]$; then,

$$
a I_{t}^{\alpha(\cdot, \cdot)} f(t)=\int_{a}^{t} \frac{1}{\Gamma[\alpha(x, t)]}(x-t)^{\alpha(x, t)-1} f(x) d x, \quad(t>a)
$$

is called the left Riemann-Liouville integral of variable fractional order $\alpha(\cdot, \cdot)$, while

$$
t I_{b}^{\alpha(\cdot, \cdot)} f(t)=\int_{t}^{b} \frac{1}{\Gamma[\alpha(x, t)]}(t-x)^{\alpha(x, t)-1} f(x) d x, \quad(t<b)
$$

is referred to as the right Riemann-Liouville integral of variable fractional order $\alpha(\cdot, \cdot)$.

Definition 2. Left and right Riemann-Liouville derivatives of variable fractional order: let $0<\alpha(x, t)<1$ for all $(x, t) \in[a, b]$. If $a I_{t}^{1-\alpha(\cdot,)} f \in A C[a, b]$, then, the left RiemannLiouville derivative of variable fractional order $\alpha(\cdot, \cdot)$ which is given as

$$
a D_{t}^{\alpha(\cdot,)} f(t)=\frac{d}{d t} \int_{a}^{t} \frac{1}{\Gamma[1-\alpha(x, t)]}(t-x)^{-\alpha(x, t)} f(x) d x
$$$$
(t>a)
$$

is called the left Riemann-Liouville derivative of variable fractional order $\alpha(\cdot, \cdot)$, while in the same line of idea we have the following expression:

$$
t D_{b}^{\alpha(\cdot,)} f(t)=\frac{d}{d t} \int_{t}^{b} \frac{1}{\Gamma[1-\alpha(x, t)]}(x-t)^{-\alpha(x, t)} f(x) d x
$$$$
(t<b)
$$

which is referred to as the right Riemann-Liouville derivative of variable fractional order $\alpha(\cdot, \cdot)$.

Definition 3. Left and right Caputo derivatives of variable fractional order: let $0<\alpha(x, t)<1$ for all $(x, t) \in[a, b]$. If $a I_{t}^{1-\alpha(\cdot, \cdot)} f \in A C[a, b]$, then, the left Riemann-Liouville derivative of variable fractional order $\alpha(\cdot, \cdot)$ is given as

$$
a D_{t}^{\alpha(\cdot,)} f(t)=\int_{a}^{t} \frac{1}{\Gamma[1-\alpha(x, t)]}(t-x)^{-\alpha(x, t)} \frac{d}{d x} f(x) d x,
$$

which is called the left Caputo derivative of variable fractional order $\alpha(\cdot, \cdot)$, while in the same line of idea we have the following expression:

$$
\begin{array}{r}
t D_{b}^{\alpha(\cdot, \cdot)} f(t)=\int_{t}^{b} \frac{1}{\Gamma[1-\alpha(x, t)]}(x-t)^{-\alpha(x, t)} \frac{d}{d x} f(x) d x, \\
(t<b)
\end{array}
$$

which is referred to as the right Caputo derivative of variable fractional order $\alpha(\cdot, \cdot)$.

However, this work we will throughout use the following definition.

2.2. Problem Formulation. The concern here is the generalization of advection dispersion equation by including a possible effect of heterogeneity or variability of the aquifer into the mathematical formulation. A one-dimensional model consisting of an infinitely long homogeneous isotropic porous medium with a steady state uniform flow with a seepage velocity $v$ is considered here. A particular chemical is injected from one end of the model for a period of time $t_{0}$ such that the input concentration varies as an exponential function of time. The value of that chemical concentration at any time $t$ and at a distance $x$ from the injection boundary, allowing for the decay and adsorption, may be obtained from the solution of the following set of equations [14]; more details for this model can be found in [15-18]. Consider

$$
D \frac{\partial^{2} C}{\partial x^{2}}-v \frac{\partial C}{\partial x}-\lambda R C=R \frac{\partial C}{\partial t}+f(x, t) .
$$

Subject to the initial and boundary conditions:

$$
C(x, 0)=0, \quad C(0, t)=c_{0} \exp (-\alpha t), \quad C_{x}(\infty, t)=0,
$$

where $D$ is the dispersion coefficient, $v$ is the seepage velocity, $R$ is the retardation factor, $\lambda$ is the radioactive decay constant, $c_{0}$ is the initial concentration, $\alpha$ is a positive constant, and $f(x, t)$ is any source and sink in the system. However, in the case of the groundwater pollution, the function $f(x, t)$ is always neglected because we assume that there is no source and sink in the system under investigation. Therefore, in our case we will set the function to be zero. In order to include explicitly the medium structure or external field changes with time into the mathematical formulation, we replace the standard derivative operator by the variational order derivative operator as follows:

$$
f(x, t)+D \frac{\partial^{2} C}{\partial x^{2}}-v \frac{\partial C}{\partial x}-\lambda R C=R D_{0}^{\alpha(t, x)}(C) .
$$

Subject to the initial and boundary conditions:

$$
C(x, 0)=0, \quad C(0, t)=c_{0} \exp (-\gamma t), \quad C_{x}(\infty, t)=0,
$$

where $\alpha(t, x)$ is a continuous function in $(0,1]$ that can be approximated from the field observation. Note that there is no analytical solution available for this new equation; therefore, our next concern is to establish the existence and uniqueness of such solution. This will be done in the next section. 


\section{Numerical Solution}

Numerical methods acquiesce estimated solutions to the overriding equation all the way through the discretization of space and time. Inside the discredited problem domain, the variable internal properties, boundaries, and stresses of the system are approximated. Deterministic, distributedparameter, and numerical models can relax the rigid idealized conditions of analytical models or lumped-parameter models, and they can therefore be more realistic and flexible for simulating fields' conditions [19-29]. The finite difference schemes for constant-order time or space fractional diffusion equations have been widely studied [19-29]. Before performing the numerical methods, we assume that (9) has a unique and sufficiently smooth solution. To establish the numerical schemes for the above equation, we let $x_{l}=l h, 0 \leq l \leq M$, $M h=L, t_{k}=k \tau, 0 \leq k \leq N$, and $N \tau=T$, and we let $h$ be the step and $\tau$ be the time size, and $M$ and $N$ be grid points.

3.1. Crank-Nicolson Scheme [30]. We bring in the CrankNicolson idea as follows. At the outset, the discretization of first- and second-order space derivative is stated as

$$
\begin{gathered}
\frac{\partial C}{\partial x}=\frac{1}{2}\left(\left(\frac{C\left(x_{l+1}, t_{k+1}\right)-C\left(x_{l-1}, t_{k+1}\right)}{2(h)}\right)\right. \\
\left.+\left(\frac{C\left(x_{l+1}, t_{k}\right)-C\left(x_{l-1}, t_{k}\right)}{2(h)}\right)\right)+O(h), \\
\frac{\partial^{2} C}{\partial x^{2}}=\frac{1}{2}\left(\left(\frac{C\left(x_{l+1}, t_{k+1}\right)-2 C\left(x_{l}, t_{k+1}\right)+C\left(x_{l-1}, t_{k+1}\right)}{(h)^{2}}\right)\right. \\
\left.+\left(\frac{C\left(x_{l+1}, t_{k}\right)-2 C\left(x_{l}, t_{k}\right)+C\left(x_{l-1}, t_{k}\right)}{(h)^{2}}\right)\right) \\
+O\left(h^{2}\right), \\
C=\frac{1}{2}\left(C\left(x_{l}, t_{k+1}\right)+C\left(x_{l}, t_{k}\right)\right) .
\end{gathered}
$$

The Crank-Nicolson scheme for the VO time fractional diffusion model can be stated as follows:

$$
\begin{aligned}
& \frac{\partial^{\alpha_{l}^{k+1}} C\left(x_{l}, t_{k+1}\right)}{\partial t_{l}^{\alpha_{l}^{k+1}}} \\
&=\frac{\tau^{-\alpha_{l}^{k+1}}}{\Gamma\left(2-\alpha_{l}^{k+1}\right)}\left(C\left(x_{l}, t_{k+1}\right)-C\left(x_{l}, t_{k}\right)\right. \\
&+\sum_{j=1}^{k}\left[C\left(x_{l}, t_{k+1-j}\right)-C\left(x_{l}, t_{k-j}\right)\right] \\
&\left.\times\left[(j+1)^{1-\alpha_{l}^{k+1}}-(j)^{1-\alpha_{l}^{k+1}}\right]\right)+O(\tau) .
\end{aligned}
$$

Now replacing (11), (12), (13), and (14) in (9) we obtain the following:

$$
\begin{aligned}
b_{l}^{k+1} & {\left[\left(C_{l+1}^{k+1}-2 C_{l}^{k+1}+C_{l-1}^{k}\right)+\left(C_{l+1}^{k}-2 C_{l}^{k}+C_{l-1}^{k}\right)\right] } \\
& +c_{l}^{k+1}\left[\left(C_{l+1}^{k+1}-C_{l-1}^{k+1}\right)+\left(C_{l+1}^{k}-C_{l-1}^{k}\right)\right] \\
& +d_{l}^{k+1}\left(C_{l}^{k+1}-C_{l}^{k}\right) \\
= & C_{l}^{k+1}-C_{l}^{k}+\sum_{i=1}^{k}\left(C_{l}^{k+1-i}-C_{l}^{k-i}\right) e_{j}^{l, k+1} \\
& +\tau^{\alpha_{l}^{k+1}} \Gamma\left(2-\alpha_{l}^{k+1}\right) f_{l}^{k+1} .
\end{aligned}
$$

Here

$$
\begin{aligned}
& b_{l}^{k+1}=\frac{D \tau_{l}^{\alpha_{l}^{k+1}} \Gamma\left(2-\alpha_{l}^{k+1}\right)}{2 R(h)^{2}}, \\
& c_{l}^{k+1}=-\frac{v \tau_{l}^{\alpha_{l}^{k+1}} \Gamma\left(2-\alpha_{l}^{k+1}\right)}{2 h R}, \\
& d_{l}^{k+1}=\frac{\lambda \tau_{l}^{\alpha_{l}^{k+1}} \Gamma\left(2-\alpha_{l}^{k+1}\right)}{2} \\
& e_{j}^{l, k+1}=(j+1)^{1-\alpha_{l}^{k+1}}-(j)^{1-\alpha_{l}^{k+1}}, p_{j}^{l, k+1}=e_{j-1}^{l, k+1}-e_{j}^{l, k+1} j=1,2, \\
& b_{l}^{1}\left[\left(C_{l+1}^{1}-2 C_{l-1}^{1}+C_{l-1}^{0}\right)+\left(C_{l+1}^{0}-2 C_{l-1}^{0}+C_{l-1}^{0}\right)\right] \\
& +c_{l}^{1}\left[\left(C_{l+1}^{1}-C_{l-1}^{1}\right)+\left(C_{l+1}^{0}-C_{l-1}^{0}\right)\right] \\
& +d_{l}^{1}\left(C_{l}^{1}-C_{l}^{0}\right)=\tau^{\alpha_{l}^{1}} \Gamma\left(2-\alpha_{l}^{1}\right) f_{l}^{1}, \\
& \text { for } k=0 \text {, } \\
& b_{l}^{k+1}\left[\left(C_{l+1}^{k+1}-2 C_{l}^{k+1}+C_{l-1}^{k}\right)+\left(C_{l+1}^{k}-2 C_{l}^{k}+C_{l-1}^{k}\right)\right] \\
& +c_{l}^{k+1}\left[\left(C_{l+1}^{k+1}-C_{l-1}^{k+1}\right)+\left(C_{l+1}^{k}-C_{l-1}^{k}\right)\right] \\
& +d_{l}^{k+1}\left(C_{l}^{k+1}-C_{l}^{k}\right) \\
& =C_{l}^{k+1}-C_{l}^{k}+\sum_{i=1}^{k}\left(C_{l}^{k+1-i}-C_{l}^{k-i}\right) e_{j}^{l, k+1} \\
& +\tau^{\alpha_{l}^{k+1}} \Gamma\left(2-\alpha_{l}^{k+1}\right) f_{l}^{k+1}, \quad \text { for } k \geq 1 .
\end{aligned}
$$

The above discretization can be rewritten in the following matrix form.

3.2. Stability Analysis of the Crank-Nicolson Scheme. In this section, we will analyze the stability conditions of the CrankNicolson scheme for the generalized advection dispersion equation. 
Let $\zeta_{l}^{k}=c_{l}^{k}-C_{l}^{k}$; here $C_{l}^{k}$ is the approximate solution at the point $\left(x_{l}, t_{k}\right),(k=1,2 \ldots, N, l=1,2, \ldots, M-1)$ and in addition $\zeta^{k}=\left[\zeta_{1}^{k}, \zeta_{2}^{k}, \ldots, \zeta_{M-1}^{k}\right]^{T}$ and the function $\zeta^{k}(x)$ is chosen to be

$$
\zeta^{k}(x)= \begin{cases}\zeta_{l}^{k} & \text { if } x_{l}-\frac{h}{2}<x \leq x_{l}+\frac{h}{2}, l=1,2, \ldots, M-1 \\ 0 & \text { if } L-\frac{h}{2}<x \leq L .\end{cases}
$$

Then, the function $\zeta^{k}(x)$ can be expressed in Fourier series as follows:

$$
\begin{gathered}
\zeta^{k}(x)=\sum_{m=-\infty}^{m=\infty} \delta_{m}(m) \exp \left[\frac{2 i \pi m k}{L}\right], \\
\delta_{k}(x)=\frac{1}{L} \int_{0}^{L} \rho^{k}(x) \exp \left[\frac{2 i \pi m x}{L}\right] d x .
\end{gathered}
$$

It was established by [25] that

$$
\left\|\zeta^{2}\right\|_{2}^{2}=\sum_{m=-\infty}^{m=\infty}\left\|\delta_{k}(m)\right\|^{2} .
$$

Observe that, for all $k, l \geq 1,0 \leq 1-\alpha_{l}^{k+1}<1$; in addition, according to the problem in point, the velocity seepage $v$, the dispersion coefficient $D$, the retardation factor $R$, and the radioactive decay constant $\lambda$ are positive constants. Then the following properties of the coefficients $c_{l}^{k+1}, d_{l}^{k+1}, p_{j}^{l, k+1}$, and $b_{l}^{k+1}$ can be established as follows.

(1) $d_{l}^{k+1}, b_{l}^{k+1}$ are positive for all $l=1,2 \ldots, M-1$.

(2) $c_{l}^{k+1}$ is negative for all $l=1,2 \ldots, M-1$.

(3) $0<e_{j}^{l, k} \leq e_{j-1}^{l, k} \leq 1$ for all $l=1,2 \ldots, M-1$.

(4) $0 \leq p_{j}^{l, k} \leq 1, \sum_{j=0}^{k-1} p_{j+1}^{l, k+1}=1-e_{k}^{l, k+1}$ for all $l=$ $1,2 \ldots, M-1$.

The error committed while approximating the solution of the generalized advection dispersion equation with CrankNicolson scheme can be presented as follows:

$$
\begin{array}{r}
b_{l}^{k+1}\left[\left(\zeta_{l+1}^{k+1}-2 \zeta_{l}^{k+1}+\zeta_{l-1}^{k+1}\right)+\left(\zeta_{l+1}^{k}-2 \zeta_{l}^{k}+\zeta_{l-1}^{k}\right)\right] \\
+c_{l}^{k+1}\left[\left(\zeta_{l+1}^{k+1}-\zeta_{l-1}^{k+1}\right)+\left(\zeta_{l+1}^{k}-\zeta_{l-1}^{k}\right)\right] \\
+d_{l}^{k+1}\left(\zeta_{l}^{k+1}-\zeta_{l}^{k}\right) \\
=\zeta_{l}^{k+1}-\zeta_{l}^{k}+\sum_{i=1}^{k}\left(\zeta_{l}^{k+1-i}-\zeta_{l}^{k-i}\right) e_{j}^{l, k+1} \\
+\tau^{\alpha_{l}^{k+1}} \Gamma\left(2-\alpha_{l}^{k+1}\right) f_{l}^{k+1} .
\end{array}
$$

If we assume that $\zeta_{l}^{k}$ in (22) can be put in the delta-exponential form as follows:

$$
\zeta_{l}^{k}=\delta_{k} \exp [i \varphi l k]
$$

where $\varphi$ is a real spatial wave number, replacing (23) in (22) we obtain

$$
\begin{aligned}
& \quad\left[1+4 b_{l}^{1} \sin ^{2}\left(\frac{\varphi h}{2}\right)-2 d_{l}^{1} \sin ^{2}\left(\frac{\varphi h}{2}\right)\right] \delta_{1} \\
& \quad=\left[1-4 b_{l}^{1} \sin ^{2}\left(\frac{\varphi h}{2}\right)-2 d_{l}^{1} \sin ^{2}\left(\frac{\varphi h}{2}\right)\right] \delta_{0}, \quad \text { for } k=0, \\
& {\left[1+4 b_{l}^{1+k} \sin ^{2}\left(\frac{\varphi h}{2}\right)-2 d_{l}^{1+k} \sin ^{2}\left(\frac{\varphi h}{2}\right)\right] \delta_{k+1}} \\
& =\left[1-4 b_{l}^{1+k} \sin ^{2}\left(\frac{\varphi h}{2}\right)-2 d_{l}^{1+k} \sin ^{2}\left(\frac{\varphi h}{2}\right)-e_{1}^{l, k+1}\right] \delta_{k} \\
& \quad+\sum_{j=0}^{k-1} p_{j+1}^{l, k+1} \delta_{k-j}+e_{k}^{l, k+1} \delta_{0}, \quad \text { for } k=1,2 \ldots, N-1 .
\end{aligned}
$$

Equation (24) can be written in the following form:

$$
\begin{gathered}
\delta_{1}=\frac{\left[1-4 b_{l}^{1} \sin ^{2}(\varphi h / 2)-2 d_{l}^{1} \sin ^{2}(\varphi h / 2)\right] \delta_{0}}{\left[1+4 b_{l}^{1} \sin ^{2}(\varphi h / 2)-2 d_{l}^{1} \sin ^{2}(\varphi h / 2)\right]}, \\
\delta_{k+1}=\left(\left[1-4 b_{l}^{1+k} \sin ^{2}\left(\frac{\varphi h}{2}\right)-2 d_{l}^{1+k} \sin ^{2}\left(\frac{\varphi h}{2}\right)-e_{1}^{l, k+1}\right] \delta_{k}\right. \\
\left.+\sum_{j=0}^{k-1} p_{j+1}^{l, k+1} \delta_{k-j}+e_{k}^{l, k+1} \delta_{0}\right) \\
\times\left[1+4 b_{l}^{1+k} \sin ^{2}\left(\frac{\varphi h}{2}\right)-2 d_{l}^{1+k} \sin ^{2}\left(\frac{\varphi h}{2}\right)\right]^{-1} .
\end{gathered}
$$

Our next concern here is to show that for all $k=1,2, \ldots, N-1$ the solution of (25) satisfies the following condition:

$$
\left|\delta_{k}\right|<\left|\delta_{0}\right| \text {. }
$$

To achieve this, we make use of the recurrence technique on the natural number $k$.

For $k=1$ and remembering that $d_{l}^{k+1}, b_{l}^{k+1}$ are positive for all $l=1,2 \ldots, M-1$, then we obtain

$$
\frac{\left|\delta_{1}\right|}{\left|\delta_{0}\right|}=\left|\frac{\left[1-4 b_{l}^{1} \sin ^{2}(\varphi h / 2)-2 d_{l}^{1} \sin ^{2}(\varphi h / 2)\right]}{\left[1+4 b_{l}^{1} \sin ^{2}(\varphi h / 2)-2 d_{l}^{1} \sin ^{2}(\varphi h / 2)\right]}\right|<1 .
$$

Assuming that for $m=2,3, \ldots, k$ the property is verified, then

$$
\begin{aligned}
\left|\delta_{k+1}\right|=\mid & \mid\left[1-4 b_{l}^{1+k} \sin ^{2}\left(\frac{\varphi h}{2}\right)-2 d_{l}^{1+k} \sin ^{2}\left(\frac{\varphi h}{2}\right)-e_{1}^{l, k+1}\right] \delta_{k} \\
& \left.+\sum_{j=0}^{k-1} p_{j+1}^{l, k+1} \delta_{k-j}+e_{k}^{l, k+1} \delta_{0}\right) \\
& \times\left[1+4 b_{l}^{1+k} \sin ^{2}\left(\frac{\varphi h}{2}\right)-2 d_{l}^{1+k} \sin ^{2}\left(\frac{\varphi h}{2}\right)\right]^{-1} \mid
\end{aligned}
$$


Making use of the triangular inequality, we obtain

$$
\begin{aligned}
\left|\delta_{k+1}\right| \leq & \left(\left|1-4 b_{l}^{1+k} \sin ^{2}\left(\frac{\varphi h}{2}\right)-2 d_{l}^{1+k} \sin ^{2}\left(\frac{\varphi h}{2}\right)-e_{1}^{l, k+1}\right|\left|\delta_{k}\right|\right. \\
& \left.+\left|\sum_{j=0}^{k-1} p_{j+1}^{l, k+1} \delta_{k-j}\right|+\left|e_{k}^{l, k+1} \delta_{0}\right|\right) \\
& \times\left|1+4 b_{l}^{1+k} \sin ^{2}\left(\frac{\varphi h}{2}\right)-2 d_{l}^{1+k} \sin ^{2}\left(\frac{\varphi h}{2}\right)\right|^{-1} .
\end{aligned}
$$

Using the recurrence hypothesis, we have

$$
\begin{aligned}
& \left|\delta_{k+1}\right| \\
& \leq\left(\left(\left|1-4 b_{l}^{1+k} \sin ^{2}\left(\frac{\varphi h}{2}\right)-2 d_{l}^{1+k} \sin ^{2}\left(\frac{\varphi h}{2}\right)\right|+\left|\sum_{j=0}^{k-1} p_{j+1}^{l, k+1}\right|\right)\right. \\
& \left.\times\left|1+4 b_{l}^{1+k} \sin ^{2}\left(\frac{\varphi h}{2}\right)-2 d_{l}^{1+k} \sin ^{2}\left(\frac{\varphi h}{2}\right)\right|^{-1}\right)\left|\delta_{0}\right|, \\
& \left|\delta_{k+1}\right| \leq\left(\frac{\left|1+4 b_{l}^{1+k} \sin ^{2}(\varphi h / 2)-2 d_{l}^{1+k} \sin ^{2}(\varphi h / 2)\right|}{\left|1+4 b_{l}^{1+k} \sin ^{2}(\varphi h / 2)-2 d_{l}^{1+k} \sin ^{2}(\varphi h / 2)\right|}\right)\left|\delta_{0}\right| \text {, } \\
& \left|\delta_{k+1}\right| \leq\left|\delta_{0}\right|
\end{aligned}
$$

and this completes the proof.

3.3. Convergence Analysis of the Crank-Nicolson Scheme. If we assume that $c\left(x_{l}, t_{k}\right)(l=1,2, \ldots, M, k=1,2 \ldots, N-1)$ is the exact solution of our problem at the point $\left(x_{l}, t_{k}\right)$, by letting $w_{l}^{k}=c\left(x_{l}, t_{k}\right)-C_{l}^{k}$ and $w^{k}=\left(0, w_{1}^{k}, w_{2}^{k}, \ldots, w_{M-1}^{k}\right)$ and substituting this in (18), we obtain

$$
\begin{gathered}
b_{l}^{1}\left[\left(w_{l+1}^{1}-2 w_{l-1}^{1}\right)\right]+c_{l}^{1}\left[\left(w_{l+1}^{1}-w C_{l-1}^{1}\right)\right]+d_{l}^{1}\left(w_{l}^{1}\right)=F_{l}^{1}, \\
\text { for } k=0, \\
b_{l}^{k+1}\left[\left(w_{l+1}^{k+1}-2 w_{l}^{k+1}+w_{l-1}^{k+1}\right)\right] \\
\quad+c_{l}^{k+1}\left[\left(w_{l+1}^{k+1}-w_{l-1}^{k+1}\right)\right]+d_{l}^{k+1} w_{l}^{k+1} \\
=F_{l}^{k+1}+\sum_{i=1}^{k}\left(w_{l}^{k-i}\right) e_{j}^{l, k+1}, \quad \text { for } k \geq 1 .
\end{gathered}
$$

Here

$$
\begin{aligned}
F_{l}^{k+1}=d_{l}^{k+1}( & \left.-C\left(x_{l}, t_{k+1}\right)+C\left(x_{l}, t_{k}\right)\right) \\
+\sum_{i=1}^{k} & \left(C\left(x_{l}, t_{k-i+1}\right)\right) e_{j}^{l, k+1} \\
& +b_{l}^{k+1}\left(-C\left(x_{l+1}, t_{k}\right)+2 C\left(x_{l}, t_{k}\right)-C\left(x_{l-1}, t_{k}\right)\right) \\
& -c_{l}^{k+1}\left(C\left(x_{l+1}, t_{k}\right)-C\left(x_{l-1}, t_{k}\right)\right) .
\end{aligned}
$$

From (11), (12), and (14),

$$
\begin{aligned}
& \frac{\partial C\left(x_{l}, t_{k+1}\right)}{\partial x}+V_{1} \\
& =\frac{1}{2}\left(\left(\frac{C\left(x_{l+1}, t_{k+1}\right)-C\left(x_{l-1}, t_{k+1}\right)}{2(h)}\right)\right. \\
& \left.+\left(\frac{C\left(x_{l+1}, t_{k}\right)-C\left(x_{l-1}, t_{k}\right)}{2(h)}\right)\right), \\
& \frac{\partial^{2} C\left(x_{l}, t_{k+1}\right)}{\partial x^{2}}+h^{2} V_{2} \\
& =\frac{1}{2}\left(\frac{\left(C\left(x_{l+1}, t_{k+1}\right)-2 C\left(x_{l}, t_{k+1}\right)+C\left(x_{l-1}, t_{k+1}\right)\right)}{h^{2}}\right. \\
& \left.+\frac{\left(C\left(x_{l+1}, t_{k}\right)-2 C\left(x_{l}, t_{k}\right)+C\left(x_{l-1}, t_{k}\right)\right)}{h^{2}}\right), \\
& \frac{\partial^{\alpha_{l}^{k+1}} C\left(x_{l}, t_{k+1}\right)}{\partial t^{\alpha_{l}^{k+1}}}+\tau V_{3} \\
& =\frac{\tau^{-\alpha_{l}^{k+1}}}{\Gamma\left(2-\alpha_{l}^{k+1}\right)}\left(C\left(x_{l}, t_{k+1}\right)-C\left(x_{l}, t_{k}\right)\right. \\
& +\sum_{j=1}^{k}\left[C\left(x_{l}, t_{k+1-j}\right)-C\left(x_{l}, t_{k-j}\right)\right] \\
& \left.\times\left[(j+1)^{1-\alpha_{l}^{k+1}}-(j)^{1-\alpha_{l}^{k+1}}\right]\right)
\end{aligned}
$$

From the above we have that

$$
F_{l}^{k+1} \leq V\left(\tau^{1+\alpha_{l}^{k+1}}+h^{2} \tau^{\alpha_{l}^{k}}+h \tau^{\alpha_{l}^{k}}\right)
$$

where $V_{1}, V_{2}, V_{3}$, and $V$ are constants. Taking into account Caputo type fractional derivative, the detailed error analysis on the above schemes can refer to the work by Diethelm et al. [31] and further work by Li and Tao [32].

Lemma 4. $\left\|w^{k+1}\right\|_{\infty} \leq V\left(\tau^{1+\alpha_{l}^{k+1}}+h^{2} \tau^{\alpha_{l}^{k}}+h \tau^{\alpha_{l}^{k}}\right)\left(e_{j}^{l, k+1}\right)^{-1}$ is true for $(k=0,1,2 \ldots, N-1)$ where $\left\|w^{k}\right\|_{\infty}=$ $\max _{1 \leq l \leq M-1}\left(w^{k}\right)$ and $V$ is a constant. In addition,

$$
\alpha^{k+1}= \begin{cases}\min _{1 \leq l \leq M-1} \alpha_{l}^{k+1}, & \text { if } \tau<1 \\ \max _{1 \leq l \leq M-1} \alpha_{l}^{k+1}, & \text { if } \tau>1\end{cases}
$$

This can be achieved via the recurrence technique on the natural number $k$. 
When $k=0$, one has the following:

$$
\begin{aligned}
\left|w_{l}^{1}\right| \leq & \left(c_{l}^{1}+b_{l}^{1}\right)\left|w_{l+1}^{1}\right| \\
& +\left(c_{l}^{1}-2 b_{l}^{1}\right)\left|w_{l-1}^{1}\right|+d_{l}^{1}\left|w_{l}^{1}\right| \\
= & \left|F_{l}^{1}\right| \\
\leq & V\left(\tau^{1+\alpha_{l}^{k+1}}+h^{2} \tau^{\alpha_{l}^{k}}+h \tau^{\alpha_{l}^{k}}\right)\left(e_{j}^{l, k+1}\right)^{-1} .
\end{aligned}
$$

Now suppose that $\left\|w^{i+1}\right\|_{\infty} \leq V\left(\tau^{1+\alpha_{l}^{i+1}}+h^{2} \tau^{\alpha_{l}^{i}}+h \tau^{\alpha_{l}^{i}}\right)\left(e_{j}^{l, i+1}\right)^{-1}$, $i=1, \ldots, N-2$. Then

$$
\begin{aligned}
& \left|w_{l}^{1+k}\right| \leq \mid b_{l}^{k+1}\left[\left(w_{l+1}^{k+1}-2 w_{l}^{k+1}+w_{l-1}^{k+1}\right)\right] \\
& +c_{l}^{k+1}\left[\left(w_{l+1}^{k+1}-w_{l-1}^{k+1}\right)\right]+d_{l}^{k+1} w_{l}^{k+1} \mid \\
& \leq\left(b_{l}^{k+1}+c_{l}^{k+1}\right)\left|w_{l+1}^{k+1}\right|+\left(b_{l}^{k+1}-c_{l}^{k+1}\right)\left|w_{l-1}^{k+1}\right| \\
& +\left(d_{l}^{k+1}-2 b_{l}^{k+1}\right)\left|w_{l}^{k+1}\right| \\
& =\left|F_{l}^{k+1}+\sum_{i=1}^{k}\left(w_{l}^{k-i}\right) e_{j}^{l, k+1}\right| \\
& \leq\left|F_{l}^{k+1}\right|+\sum_{i=1}^{k}\left|w_{l}^{k-i}\right| e_{j}^{l, k+1} \\
& \leq V\left(\tau^{1+\alpha_{l}^{k+1}}+h^{2} \tau^{\alpha_{l}^{k}}+h \tau^{\alpha_{l}^{k}}\right)+\sum_{i=1}^{k}\left\|w_{l}^{k-i}\right\|_{\infty} e_{j}^{l, k+1} \\
& \leq V\left(\tau^{1+\alpha_{l}^{k+1}}+h^{2} \tau^{\alpha_{l}^{k}}+h \tau^{\alpha_{l}^{k}}\right) \\
& \times\left(e_{j}^{l, k+1}+e_{0}^{l, k+1}-e_{j}^{l, k+1}\right)\left(e_{j}^{l, k+1}\right)^{-1} \\
& \leq V\left(\tau^{1+\alpha_{l}^{k+1}}+h^{2} \tau^{\alpha_{l}^{k}}+h \tau^{\alpha_{l}^{k}}\right)\left(e_{0}^{l, k+1}\right)\left(e_{j}^{l, k+1}\right)^{-1}, \\
& \left|w_{l}^{1+k}\right| \leq V\left(\tau^{1+\alpha_{l}^{k+1}}+h^{2} \tau^{\alpha_{l}^{k}}+h \tau^{\alpha_{l}^{k}}\right)\left(e_{j}^{l, k+1}\right)^{-1} \text {. }
\end{aligned}
$$

This completes the proof.

Theorem 5. The Crank-Nicolson scheme is convergent, and there exists a positive constant $V$ such that

$$
\begin{array}{r}
\quad\left|C_{l}^{k}-c\left(x_{l}, t_{k}\right)\right| \leq V\left(\tau+h+h^{2}\right), \\
l=1,2, \ldots, M-1, \quad k=1,2, \ldots, N .
\end{array}
$$

An interested reader can find the solvability of the CrankNicholson scheme in the work done by [33]. Therefore, the details of the proof will not be presented in this paper.

\section{Numerical Simulations}

In this section, we present the numerical solutions of the following three cases.
TABLE 1: Theoretical parameters.

\begin{tabular}{lcccccc}
\hline Parameters & $D$ & $v$ & $R$ & $\gamma$ & $c_{0}$ & $\lambda$ \\
\hline Values & 2 & 2 & 1 & 0.86 & 100 & 1 \\
\hline
\end{tabular}

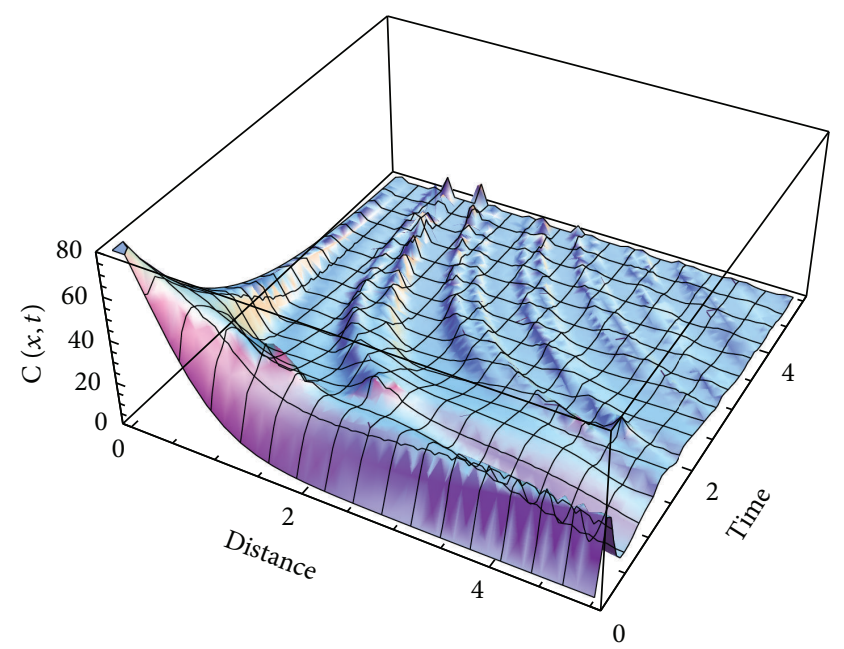

FIGURE 1: Approximate solution of the modified equation as function of time and space.

The case where we assume that the variation of the geological formation in which the contamination is migrating can be modelled as function of time and space as $\alpha(x, t)$. To include explicitly this variability in the mathematical formulation, we reformulate the hydrodynamic dispersion equation as follows:

$$
\begin{gathered}
D \frac{\partial^{2} C}{\partial x^{2}}-v \frac{\partial C}{\partial x}-\lambda R C=R D_{0}^{\alpha(t, x)}(C), \\
\alpha(x, t)=1-\sin (x t), \\
C(x, 0)=0, \quad C(0, t)=c_{0} \exp (-\gamma t) .
\end{gathered}
$$

The theoretical parameters used in this simulation are given in Table 1.

Figure 1 shows the approximate solution of the main problem as function of space and time. Figure 2 shows the density plot of the approximate solution of the main problem. Figure 3 shows the variation of the concentration as function of time for a fixed space. Figures 1 and 3 show the solution of the variation order advection and advection equation, respectively, and Figure 2 shows the contour map of variational order advection equation.

\section{Discussion and Conclusions}

Natural geological deposits with highly contrasting permeability may form mobile and relatively immobile zones, where the potential mass exchange between mobile and immobile zones results in a wide time distribution for solute "trapping." The transport process, groundwater, is, by its very nature, always in contact with the matrix of an aquifer. There is thus a possibility that the solutes may interact with the rock 


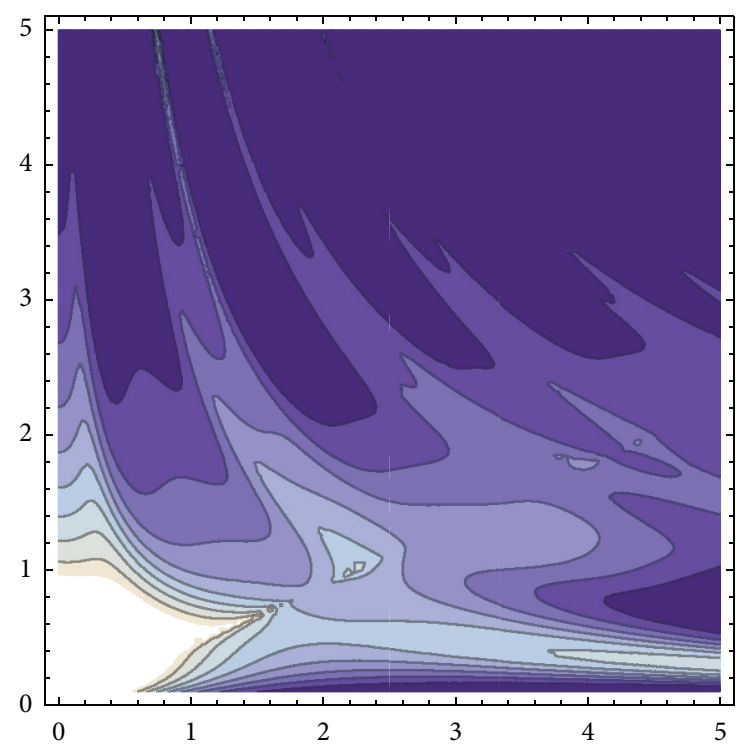

FIGURE 2: Solution of the modified equation seing as contour map.

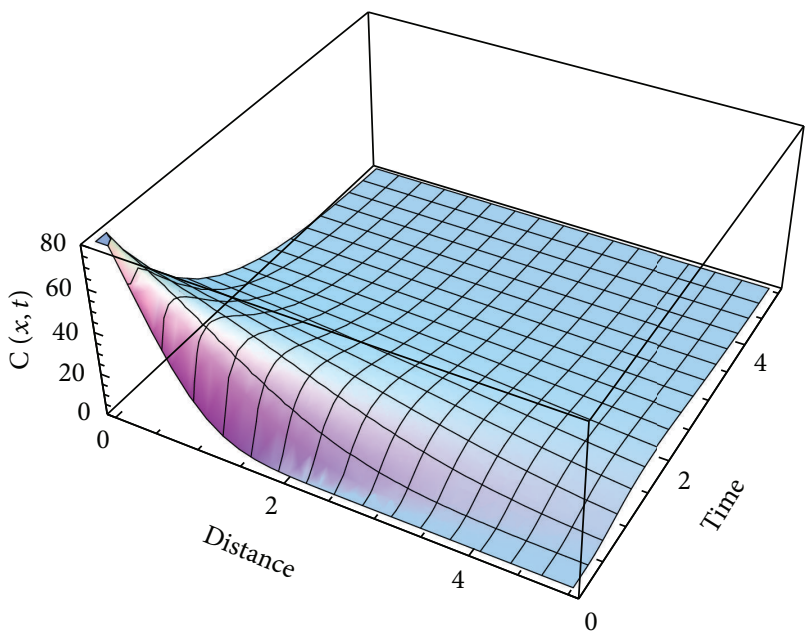

FIGURE 3: Solution of the standard equation.

matrix and one another. A true mathematical model for groundwater pollution must therefore be able to account for interactions between the dissolved solids and matrix of the aquifer. It will thus be advantageous to look at the nature of the interactions between dissolved solids and a porous medium that may be expected in groundwater pollution. Experimental evidence indicates that when a dissolved solid comes in contact with the matrix of a porous medium it may (a) pass through the medium with no apparent effect, (b) be absorbed by the porous matrix, and (c) react with the porous matrix and other substances dissolved in the fluid. The dissolved solids encountered in porous flow are, for this reason, often classified as conservative, nonconservative, and reactive tracers. This behaviour implies that the quantity of dissolved solids in a porous medium depends not only on the flow pattern but also on the nature of the porous matrix and the solution. These situations (a), (b), and (c) can be characterized efficiently by the time-nonlocal model, including the time variational order advection dispersion equation. If the high-permeable material tends to form preferential flow paths, such as the interconnected paleochannels observed in alluvial depositional systems, then the solute transport may show a heavy leading edge, which can be described by the Variational Order Advection Dispersion Equation (VOADE). Development of partial differential equations such as the advection-dispersion equation (ADE) begins with assumptions about the random behaviour of a single particle: possible velocities it may experience in a flow field and the length of time it may be immobilized. When assumptions underlying the ADE are relaxed, a fractional ADE (FADE) can arise, with a non-integer-order derivative on time or space terms. Fractional ADEs are nonlocal; they describe transport affected by hydraulic conditions at a distance. Space fractional ADEs arise when velocity variations are heavy tailed and describe particle motion that accounts for variation in the flow field over the entire system [34]. But when the geological formation, under which this pollution moves, is changing in time and space, the VOADE can be used. Many other uses of the variable-order derivatives can be found in the following works [35-40].

\section{Conflict of Interests}

The authors declare that there is no conflict of interests regarding the publication of this paper.

\section{Acknowledgement}

This work was partially financially supported by the Leon Claude Fellowships of 2014 of South Africa. Adem Kilicman gratefully acknowledges that this research was partially supported by the University Putra Malaysia under the Grant Scheme GB-IBT having the project number GBIBT/2013/9420100.

\section{References}

[1] T. H. Solomon, E. R. Weeks, and H. L. Swinney, "Observation of anomalous diffusion and Lévy flights in a two-dimensional rotating flow," Physical Review Letters, vol. 71, no. 24, pp. 39753978, 1993.

[2] S. Bhalekar, V. Daftardar-Gejji, D. Baleanu, and R. Magin, "Fractional Bloch equation with delay," Computers \& Mathematics with Applications, vol. 61, no. 5, pp. 1355-1365, 2011.

[3] R. L. Magin, Fractional Calculus in Bioengineering, Begell House Publisher, Redding, Conn, USA, 2006.

[4] R. L. Magin, O. Abdullah, D. Baleanu, and X. J. Zhou, "Anomalous diffusion expressed through fractional order differential operators in the Bloch-Torrey equation," Journal of Magnetic Resonance, vol. 190, no. 2, pp. 255-270, 2008.

[5] A. V. Chechkin, R. Gorenflo, and I. M. Sokolov, "Fractional diffusion in inhomogeneous media," Journal of Physics, vol. 38, no. 42, pp. L679-L684, 2005. 
[6] F. Santamaria, S. Wils, E. de Schutter, and G. J. Augustine, "Anomalous diffusion in Purkinje cell dendrites caused by spines," Neuron, vol. 52, no. 4, pp. 635-648, 2006.

[7] H. Sun, W. Chen, and Y. Q. Chen, "Variable-order fractional differential operators in anomalous diffusion modeling," Physica A, vol. 388, no. 21, pp. 4586-4592, 2009.

[8] H. Sun, Y. Q. Chen, and W. Chen, "Random-order fractional differential equation models," Signal Processing, vol. 91, no. 3, pp. 525-530, 2011.

[9] Y. Q. Chen and K. L. Moore, "Discretization schemes for fractional-order differentiators and integrators," IEEE Transactions on Circuits and Systems I, vol. 49, no. 3, pp. 363-367, 2002.

[10] S. Umarov and S. Steinberg, "Variable order differential equations with piecewise constant order-function and diffusion with changing modes," Zeitschrift für Analysis und ihre Anwendungen, vol. 28, no. 4, pp. 431-450, 2009.

[11] B. Ross and S. Samko, "Fractional integration operator of variable order in the Hölder spaces $H^{\lambda(x)}$," International Journal of Mathematics and Mathematical Sciences, vol. 18, no. 4, pp. 777-788, 1995.

[12] H. T. C. Pedro, M. H. Kobayashi, J. M. C. Pereira, and C. F. M. Coimbra, "Variable order modeling of diffusive-convective effects on the oscillatory flow past a sphere," Journal of Vibration and Control, vol. 14, no. 9-10, pp. 1659-1672, 2008.

[13] D. Ingman and J. Suzdalnitsky, "Application of differential operator with servo-order function in model of viscoelastic deformation process," Journal of Engineering Mechanics, vol. 131, no. 7, pp. 763-767, 2005.

[14] R. W. Cleary and M. J. Ungs, Groundwater Pollution and Hydrology. Mathematical Models and Computer Programs, vol. 08540, Water Resources Programs, Princeton University, Princeton, NJ, USA, 1978.

[15] M. T. van Genuchten and W. J. Alves, "Analytical solutions of one dimensional convective-dispersive solute transport equations," United States Department of Agriculture, no. 1661, 1982.

[16] D. K. Jaiswal, A. Kumar, N. Kumar, and R. R. Yadav, "Analytical solutions for temporally and spatially dependent solute dispersion of pulse type input concentration in one-dimensional semi-infinite media," Journal of Hydro-Environment Research, vol. 2, no. 4, pp. 254-263, 2009.

[17] D. K. Jaiswal, A. Kumar, N. Kumar, and M. K. Singh, "Solute transport along temporally and spatially dependent flows through horizontal semi-infinite media: dispersion proportional to square of velocity," Journal of Hydrologic Engineering, vol. 16, no. 3, pp. 228-238, 2011.

[18] R. R. Yadav, D. K. Jaiswal, and G. Gulrana, "Two-dimensional solute transport for periodic flow in isotropic porous media: an analytical solution," Hydrological Processes, vol. 26, no. 22, pp. 3425-3433, 2012.

[19] A. Atangana and S. C. Oukouomi Noutchie, "Stability and convergence of a time-fractional variable order Hantush equation for a deformable aquifer," Abstract and Applied Analysis, vol. 2013, Article ID 691060, 8 pages, 2013.

[20] Y. Zhang, "A finite difference method for fractional partial differential equation," Applied Mathematics and Computation, vol. 215, no. 2, pp. 524-529, 2009.

[21] C. Tadjeran, M. M. Meerschaert, and H. P. Scheffler, "A secondorder accurate numerical approximation for the fractional diffusion equation," Journal of Computational Physics, vol. 213, no. 1, pp. 205-213, 2006.
[22] M. M. Meerschaert and C. Tadjeran, "Finite difference approximations for fractional advection-dispersion flow equations," Journal of Computational and Applied Mathematics, vol. 172, no. 1, pp. 65-77, 2004.

[23] W. H. Deng, "Numerical algorithm for the time fractional Fokker-Planck equation," Journal of Computational Physics, vol. 227, no. 2, pp. 1510-1522, 2007.

[24] C. P. Li, A. Chen, and J. Ye, "Numerical approaches to fractional calculus and fractional ordinary differential equation," Journal of Computational Physics, vol. 230, no. 9, pp. 3352-3368, 2011.

[25] C. M. Chen, F. Liu, I. Turner, and V. Anh, "A Fourier method for the fractional diffusion equation describing sub-diffusion," Journal of Computational Physics, vol. 227, no. 2, pp. 886-897, 2007.

[26] S. B. Yuste and L. Acedo, "An explicit finite difference method and a new von Neumann-type stability analysis for fractional diffusion equations," SIAM Journal on Numerical Analysis, vol. 42, no. 5, pp. 1862-1874, 2005.

[27] I. Podlubny, A. Chechkin, T. Skovranek, Y. Chen, and B. M. Vinagre Jara, "Matrix approach to discrete fractional calculus. II. Partial fractional differential equations," Journal of Computational Physics, vol. 228, no. 8, pp. 3137-3153, 2009.

[28] A. Atangana, "On the solution of an acoustic wave equation with variable-order derivative loss operator," Advances in Difference Equations, vol. 2013, article 167, 2013.

[29] R. Lin, F. Liu, V. Anh, and I. Turner, "Stability and convergence of a new explicit finite-difference approximation for the variable-order nonlinear fractional diffusion equation," Applied Mathematics and Computation, vol. 212, no. 2, pp. 435-445, 2009.

[30] J. Crank and P. Nicolson, "A practical method for numerical evaluation of solutions of partial differential equations of the heat-conduction type," vol. 43, pp. 50-67, 1947.

[31] K. Diethelm, N. J. Ford, and A. D. Freed, "Detailed error analysis for a fractional Adams method," Numerical Algorithms, vol. 36, no. 1, pp. 31-52, 2004.

[32] C. P. Li and C. X. Tao, "On the fractional Adams method," Computers \& Mathematics with Applications, vol. 58, no. 8, pp. 1573-1588, 2009.

[33] M. Cui, "Compact finite difference method for the fractional diffusion equation," Journal of Computational Physics, vol. 228, no. 20, pp. 7792-7804, 2009.

[34] A. Atangana, A generic assessment of waste disposal at Douala city: principle, practices and uncertainties [Ph.D. thesis], University of the Free State South Africa, Bloemfontein, South Africa, 2012.

[35] D. Valério and J. Sá da Costa, "Variable-order fractional derivatives and their numerical approximations," Advances in Fractional Signals and Systems, vol. 91, no. 3, pp. 470-483, 2011.

[36] D. Valério, G. Vinagre, J. Domingues, and J. Sá da Costa, "Variable-order fractional derivatives and their numerical approximations I-real orders," in Symposium on Fractional Signals and Systems, Caparica, 2009.

[37] D. Valério, G. Vinagre, J. Domingues, and J. Sá da Costa, "Variable-order fractional derivatives and their numerical approximations II-complex orders," in Symposium on Fractional Signals and Systems, Caparica, 2009.

[38] A. Razminia, A. F. Dizaji, and V. J. Majd, “Solution existence for non-autonomous variable-order fractional differential equations," Mathematical and Computer Modelling, vol. 55, no. 3-4, pp. 1106-1117, 2012. 
[39] S. Ma, Y. Xu, and W. Yue, "Numerical solutions of a variableorder fractional financial system," Journal of Applied Mathematics, vol. 2012, Article ID 417942, 14 pages, 2012.

[40] H. Sheng, H. Sun, Y. Chen, and T. Qiu, "Synthesis of multifractional Gaussian noises based on variable-order fractional operators," Signal Processing, vol. 91, no. 7, pp. 1645-1650, 2011. 


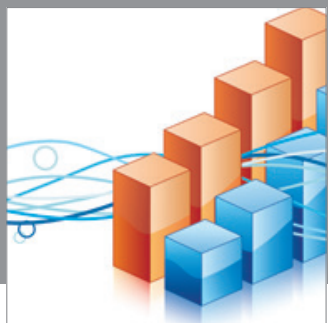

Advances in

Operations Research

mansans

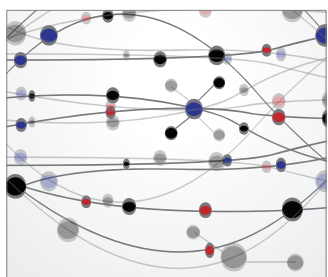

The Scientific World Journal
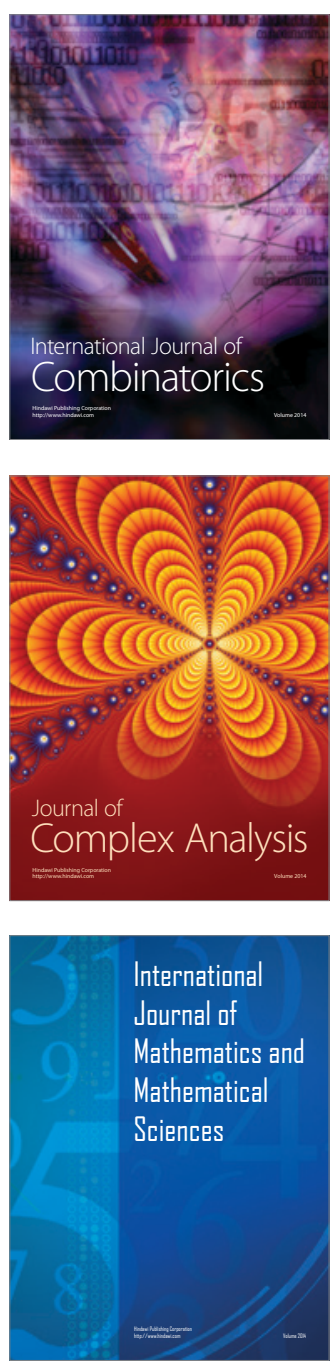
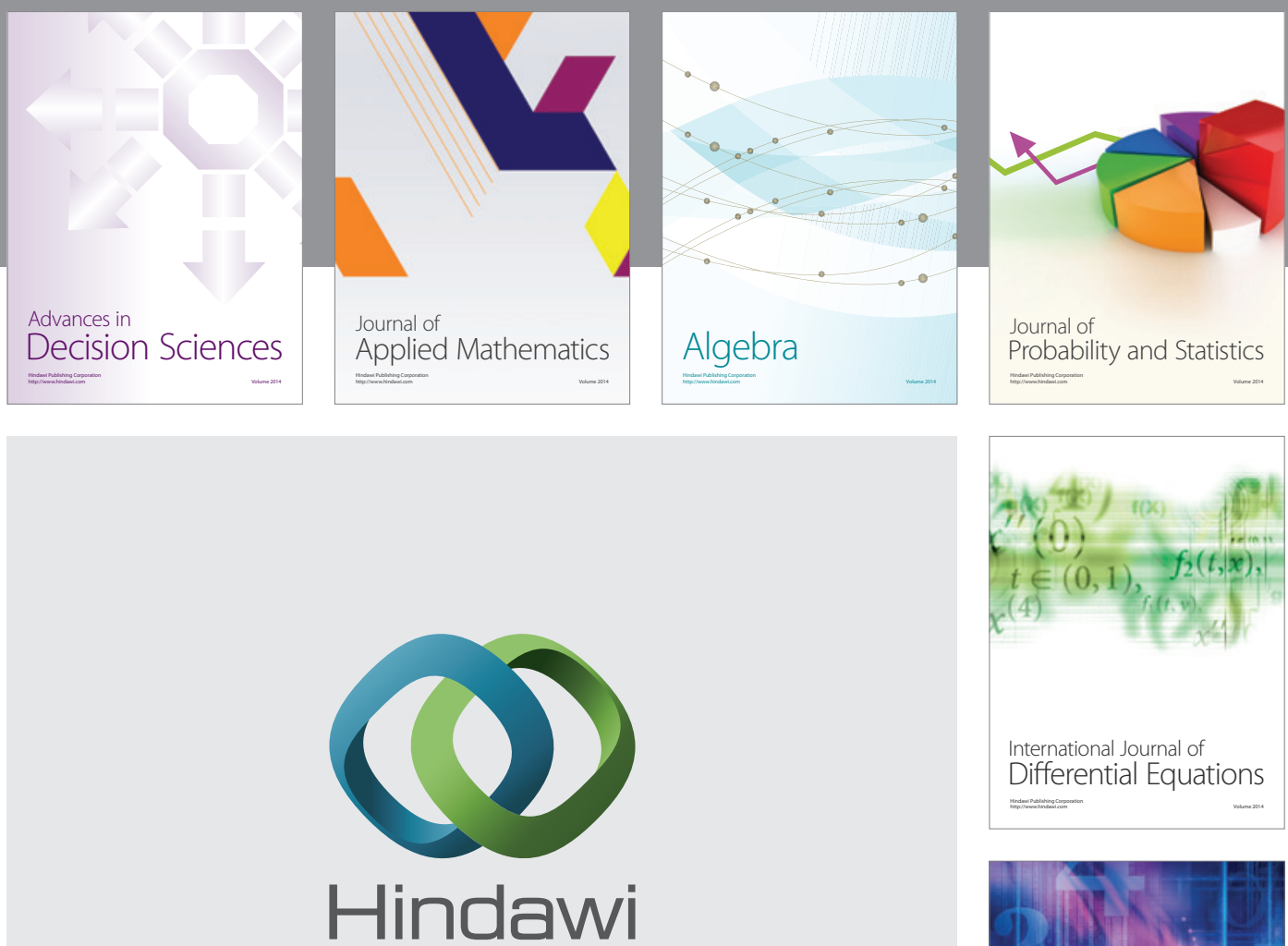

Submit your manuscripts at http://www.hindawi.com
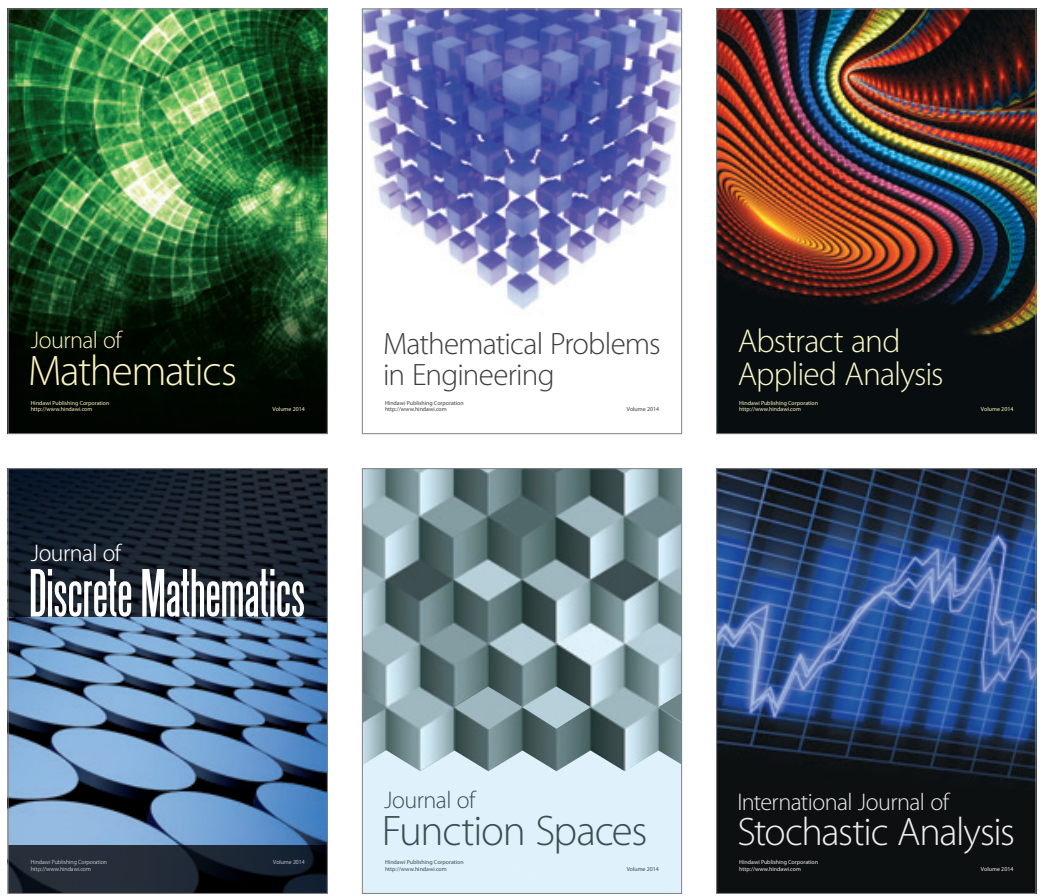

Journal of

Function Spaces

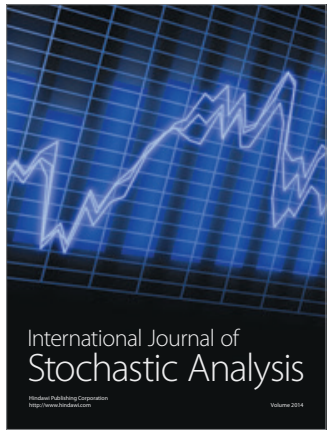

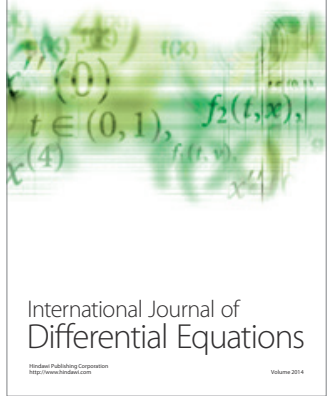
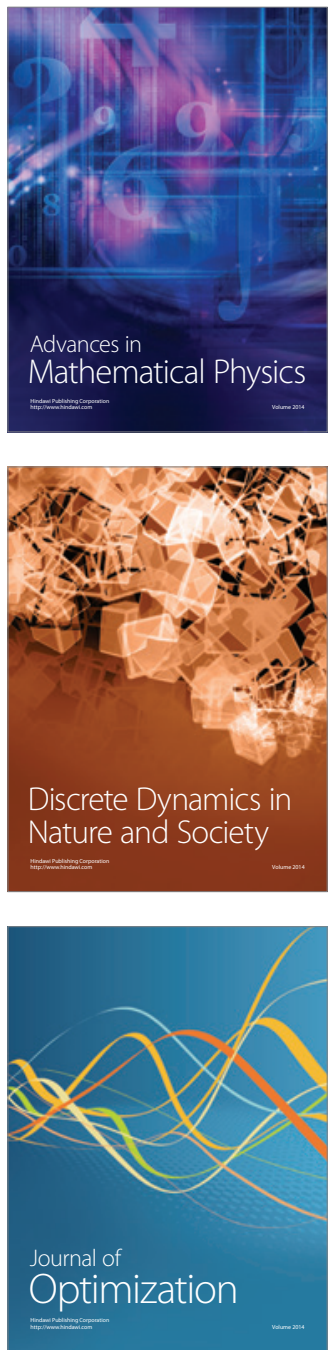